\title{
Identifying Representative Synergy Matrices for Describing Muscular Activation Patterns During Multidirectional Reaching in the Horizontal Plane
}

\author{
Silvia Muceli, ${ }^{1}$ Andreas Trøllund Boye, ${ }^{1}$ Andrea d'Avella, ${ }^{2}$ and Dario Farina ${ }^{1}$ \\ ${ }^{1}$ Center for Sensory-Motor Interaction, Department of Health Science and Technology, Aalborg University, Aalborg, Denmark; \\ and ${ }^{2}$ Department of Neuromotor Physiology, Santa Lucia Foundation, Rome, Italy
}

Submitted 30 June 2009; accepted in final form 12 January 2010

Muceli S, Boye AT, d'Avella A, Farina D. Identifying representative synergy matrices for describing muscular activation patterns during multidirectional reaching in the horizontal plane. J Neurophysiol 103: 1532-1542, 2010. First published January 13, 2010; doi:10.1152/jn.00559.2009. Muscle synergies have been proposed as a simplifying principle of generation of movements based on a low-dimensional control by the CNS. This principle may be useful for movement restoration by, e.g., functional electrical stimulation (FES), if a limited set of synergies can describe several functional tasks. This study investigates the possibility of describing a multijoint reaching task of the upper limb by a linear combination of one set of muscle synergies common to multiple directions. Surface electromyographic (EMG) signals were recorded from 12 muscles of the dominant upper limb of eight healthy men during single-joint movements and a multijoint reaching task in 12 directions in the horizontal plane. The movement kinematics was recorded by a motion analysis system. Muscle synergies were extracted with nonnegative matrix factorization of the EMG envelopes. Synergies were computed either from the single-joint movements to describe the two degrees of freedom independently or from the multijoint movements. On average, the multijoint reaching task could be accurately described in all the directions (coefficient of determination $>0.85$ ) by a linear combination of either four synergies extracted from the individual degrees of freedom or three synergies extracted from multijoint movements in at least three reaching directions. These results indicate that a large set of multijoint movements can be generated by a synergy matrix of limited dimensionality and common to all directions if the synergies are extracted from a representative number of directions. The linear combination of synergies may thus be used in strategies for restoring functions, such as FES.

\section{N T R O D U C T I O N}

The planning and execution of movements involve the control of several nonlinear actuators (muscles) operating in an ever changing environment. It would be computationally very complex for the CNS to control each muscle individually to generate a movement (Bernstein 1967). Thus the control of movement by the CNS may be based on an internal lowdimensional task-level representation of the external environment. According to this concept, movements are executed by translating the task-level commands into muscle activation patterns (Kandel et al. 2000; Ting and McKay 2007). Synchronous muscle synergies have been suggested as a model for this translation (Bizzi et al. 2002; d'Avella and Bizzi 2005; Ivanenko et al. 2004; Saltiel et al. 2001; Ting and Macpherson 2005; Tresch et al. 1999; Weiss and Flanders 2004).

Address for reprint requests and other correspondence: D. Farina, Center for Sensory-Motor Interaction (SMI), Department of Health Science and Technology, Aalborg University, Fredrik Bajers Vej 7 D-3, DK-9220 Aalborg, Denmark (E-mail: df@hst.aau.dk).
A muscle synergy is a set of relative nonnegative levels of muscle activation (Ting and McKay 2007) and each synergy is activated by a nonnegative activation signal. The absolute activation level of each muscle over time is the summation of the contributions from all synergies, weighted by the activation signals. The concept of muscle synergies is supported by results of studies at different levels of the neuromuscular system. For example, a common drive in the oscillation of discharge rates of motor units has been observed across synergistic muscles (DeLuca and Erim 2002). Moreover, there is a correlation between the discharge of neurons located in the primary motor cortex of the monkey and surface electromyographs (EMG) of multiple muscles, suggesting that descending fibers project to motor neuron pools of different muscles (Holdefer and Miller 2002). Similar results were obtained in studies in which cortical neurons of the cat (Ethier et al. 2006) or interneuronal sites in the spinal cord of the frog were stimulated (Saltiel et al. 2001). These results suggest that motor control by the CNS may be organized into a small number of modules that can be activated by descending neurons and combined to produce a wide range of movements.

Matrix factorization algorithms have been proposed for the decomposition of a muscle activity pattern into synergies and activation signals. These algorithms include principal component analysis (PCA), factor analysis (FA), independent component analysis (ICA), and nonnegative matrix factorization (NMF) (Tresch et al. 2006).

The synergistic model of motor control is attractive for designing strategies for movement restoration. According to the concept of synergistic muscle activations, the recruitment of a large number of muscles may be performed by a reduced number of independent control signals (activation signals) that are translated into individual muscular activation patterns by a transformation matrix (the synergy matrix). These activation signals might be delivered either through intraspinal electrical stimulation, exploiting the intrinsic synergistic organization of the spinal circuitries, or by functional electrical stimulation (FES) of peripheral nerves that control specific muscles, using an appropriately derived synergy matrix. The first approach has been widely investigated in theoretical (Barbeau et al. 1999; Mussa-Ivaldi 1992; Mussa-Ivaldi and Giszter 1992) and experimental studies (Bizzi et al. 1991; Giszter et al. 1993; Lemay and Grill 2004; Lemay et al. 2001; Mussa-Ivaldi et al. 1994). Moreover, it has been shown that the spinal motor output modularity is preserved in the cat after chronic spinalization (Boyce and Lemay 2009), suggesting the possibility of using intraspinal microstimulation to restore motor output following chronic spinal cord injury. 
The possibility of using synergistic control in function restoration using FES of peripheral nerves has been less extensively explored. Such an approach requires the identification of a synergy matrix that can accurately describe a large number of functional tasks. Although it has been shown that some synergies extracted from different movements are similar to each other (d'Avella and Bizzi 2005), the possibility that a single matrix of limited dimensionality can describe (and thus predict) a large variety of tasks (including tasks not used for the computation of the matrix) has been examined in only a few studies (Ajiboye and Weir 2009; Torres-Oviedo et al. 2006).

In this study, we analyzed the multijoint system consisting of the shoulder and elbow during movements in the horizontal plane. The functional degrees of freedom (DOFs) in these movements are two, one for each joint ( $\mathrm{Li}$ 2006). This system is relevant in many functional tasks and has been investigated in several rehabilitation strategies for recovery of function (e.g., Popovic et al. 2002). Recent studies on FES of the complex shoulder-elbow have focused on the identification of a subset of muscles under voluntary control from which stimulation levels necessary for paralyzed muscles can be predicted (Hincapie and Kirsch 2007, 2009). This approach is consistent with the notion that muscles across the upper extremity are controlled by the CNS in a synergistic manner. Therefore a linear controller based on muscle synergies should in principle allow obtaining performance comparable to that of a fulldimension, nonlinear controller (Berniker et al. 2009).

This study is intended to provide additional support to the use of a synergistic controller in FES by proposing a method to effectively reduce the number of control signals. We hypothesize that one synergy matrix of limited dimensionality can describe the muscle activation of all functional reaching tasks in the horizontal plane and we propose strategies to extract these context-unspecific synergies. Therefore the aim of the study was to demonstrate that one synergy matrix is sufficient to describe all functionally relevant reaching tasks in the horizontal plane and to propose methods to experimentally compute this synergy matrix. The results of the study have direct relevance in designing electrical stimulation patterns for paralyzed muscles.

\section{METHODS}

\section{Subjects}

Eight healthy men (age, $26.6 \pm 3.2 \mathrm{yr}$; weight, $75.8 \pm 8.2 \mathrm{~kg}$; stature, $180.1 \pm 7.6 \mathrm{~cm})$ volunteered for the experiments. Subjects provided written informed consent before participation and the procedures were approved by the local ethics committee.

\section{Procedures}

The experiment consisted of movements in the horizontal plane. The subjects sat in front of a table with the right (dominant) arm supported by a custom-designed manipulandum that allowed flexion and extension of the shoulder and elbow (Fig. 1A). The upper trunk was not restrained, but analysis of the kinematics data showed that its movement during the investigated tasks was negligible, as previously observed (Galloway and Koshland 2002). "Reaching" in the horizontal plane was defined as the task of moving the arm such that the position of the wrist, which was projected on the table surface by a laser beam, was within a circle of $20-\mathrm{mm}$ diameter (target). Audible

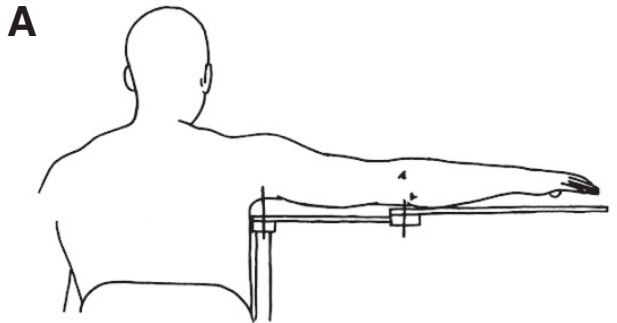

B
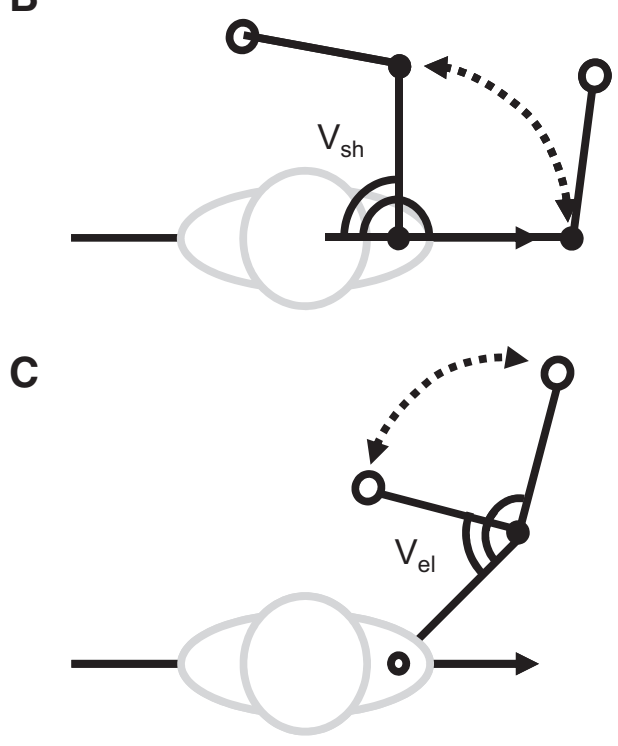

D

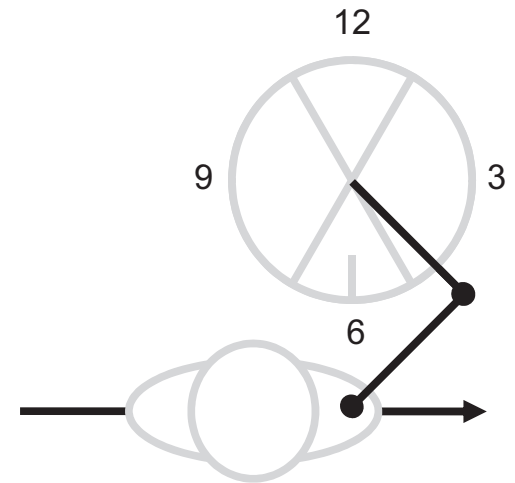

FIG. 1. A: posterior view of the manipulandum used to perform single- and multijoint tasks in the horizontal plane. $B$ : shoulder extension-flexion movements. The initial position corresponded to the shoulder flexed at $\mathrm{V}_{\mathrm{sh}}=90^{\circ}$ and the final position corresponded to the shoulder extended at $\mathrm{V}_{\mathrm{sh}}=180^{\circ}$. This task was repeated for 3 elbow angles $\left(\mathrm{V}_{\mathrm{el}}=60,105,150^{\circ}\right)$. $C$ : elbow extension-flexion movements. The initial position corresponded to the elbow flexed at $\mathrm{V}_{\mathrm{el}}=60^{\circ}$ and the final position corresponded to the elbow extended at $\mathrm{V}_{\mathrm{el}}=150^{\circ}$. This task was repeated for 3 shoulder angles $\left(\mathrm{V}_{\mathrm{sh}}=90,135\right.$, $\left.180^{\circ}\right)$. $D$ : reaching tasks with starting point the center of the circle and targets around the circle. The reaching tasks were repeated for 3 positions of the center point.

cues were given to indicate the start and end of a movement and the subjects trained for the tasks for about 15 min before the recordings.

\section{Tasks}

Each movement had a duration of $1 \mathrm{~s}$ and movements were separated by $5 \mathrm{~s}$ of rest. Synchronous synergies are invariant for speed and load both in terms of muscle activations (Gabriel 1997), joint 
torque (Gottlieb et al. 1996), and joint angles (Lacquaniti and Soechting 1982) and thus speed and load were not varied in this study. The subjects performed single-joint movements of the shoulder and elbow and multidirectional, multijoint reaching movements in the horizontal plane. The order with which the movements were performed was randomized.

For single-joint movements with the shoulder, the initial position corresponded to the shoulder flexed at $90^{\circ}$ and the elbow at an angle that varied among $60^{\circ}(\mathrm{SH} 1), 105^{\circ}(\mathrm{SH} 2)$, or $150^{\circ}(\mathrm{SH} 3)(\mathrm{Fig} .1 B)$. The subject extended the shoulder from the initial position to $180^{\circ}$ of flexion in $1 \mathrm{~s}$, rested for $5 \mathrm{~s}$, returned to the starting position in $1 \mathrm{~s}$ by flexing the shoulder, and rested in the starting position for $5 \mathrm{~s}$ before the next movement. The elbow was fixed at one of the three angles during these tasks. The shoulder extension/flexion task was repeated 10 times, which took $120 \mathrm{~s}$, for each position of the elbow.

Single-joint movements of the elbow were performed in a way similar to that of the shoulder movements (Fig. 1C). The initial position corresponded to the elbow flexed at $60^{\circ}$ and the shoulder joint at $90^{\circ}(\mathrm{EL} 1), 135^{\circ}(\mathrm{EL} 2)$, or $180^{\circ}$ (EL3). The target position corresponded to the elbow at $150^{\circ}$. The number of repetitions and resting intervals was the same as that for the shoulder tasks.

The multijoint movements in the horizontal plane consisted in reaching 12 targets evenly spaced along a circumference (Fig. 1D). The radius of the circumference was half the distance between the olecranon and styloid process of the ulna. The starting point was the center of the circle and corresponded to the elbow joint angle at $90^{\circ}$ and the shoulder joint angle at either $100^{\circ}(\mathrm{JO} 1), 120^{\circ}(\mathrm{JO} 2)$, or $140^{\circ}$ (JO3). Each target was reached once in random sequence. The reaching movement had 1 -s duration and was followed by $5 \mathrm{~s}$ of rest at the target position and the return to the center point in $1 \mathrm{~s}$. Thus reaching all targets took $144 \mathrm{~s}$ and was repeated for the three initial shoulder angles.

\section{EMG recordings}

Surface EMG signals were recorded in bipolar derivations with pairs of $\mathrm{Ag} / \mathrm{AgCl}$ electrodes (Ambu Neuroline 720 01-K/12; Ambu, Ballerup, Denmark) with $22 \mathrm{~mm}$ of center-to-center spacing. Prior to electrode placement the skin was shaved, if necessary, and lightly abraded. The EMG signals were amplified with a gain of 2,000 (EMG-USB, LISiN; OT Bioelettronica, Turin, Italy), band-pass filtered (eighth-order Bessel filter, bandwidth 10-750 Hz), sampled at $2,048 \mathrm{~Hz}$, and A/D converted on 12 bits. A reference electrode was placed at the wrist of the nondominant arm.

The EMG signals were recorded from the following muscles [electrode locations are in accord with those reported by Hermens et al. (1999) unless specified otherwise]: brachioradialis (BIO, one sixth the distance ranging from the midpoint between the cubit fossa and the lateral epicondyle to the styloid process of ulna), anconeus (ANC, $2 \mathrm{~cm}$ distal to the midpoint between the lateral epicondyle and the olecranon process), biceps brachii medial head (BME), biceps brachii lateral head (BLA), brachialis (BIA, $4 \mathrm{~cm}$ proximal with respect to the midpoint between the fossa cubit and the lateral epicondyle), triceps lateral head (TLA), triceps long head (TLO), deltoid medial part (DME), pectoralis major (PEC, one third the distance ranging from the glenohumeral joint to the lowest point of the sternum), deltoid anterior part (DAN), deltoid posterior part (DPO), and latissimus dorsi (LAT, $4 \mathrm{~cm}$ below the inferior border of the scapula, half the distance between the spine and the lateral edge of the body).

\section{Kinematics}

Reflective ball-shaped markers (18-mm diameter) were placed in correspondence of the left acromion (LAC), right acromion (RAC), lateral epicondyle of humerus (LEP), and posterior midpoint between the styloid processes of radius and ulna (MST). Positions of the markers were tracked with a motion analysis system (Qualisys Track
Manager; Qualisys, Gothenburg, Sweden) with eight infrared digital video cameras (ProReflex MCU; Qualisys). The kinematics data were recorded with a sampling frequency of $240 \mathrm{~Hz}$ and synchronized with the EMG recordings for data analysis.

\section{Synergy model}

It was assumed that a system with $D$ biomechanical DOFs is driven by a muscular system with $N$ activation signals, denoted by the vector $P(k)$

$$
P(k)=\left[p_{1}(k), p_{2}(k), \ldots, p_{N}(k)\right]^{T}
$$

where $p_{n}(k)$ is the $n$th activation signal and $k$ indicates the time. In this model, each activation signal represents a synaptic input from premotor neurons to motor neuron pools. Activations signals will also be referred to as control signals in the context of electrically stimulated muscles.

Assuming that $M$ muscles are involved in the movement, their activity levels at the time instant $k$ are described by the muscle activation vector $X(k)$

$$
X(k)=\left[x_{1}(k), x_{2}(k), \ldots, x_{M}(k)\right]^{T}
$$

where $x_{m}(k)$ is the level of activation of the $m$ th muscle. The activation of a muscle represents the combined output of all active motor neurons innervating the muscle. In this study, $X(k)$ was estimated from rectified and low-pass filtered surface EMG signals, assuming that cross talk was negligible. The evolution of the muscle activation vector over time is referred to as muscle activation pattern.

The relationship between $X(k)$ and $P(k)$ is described as

$$
X(k) \approx X_{r}(k)=S \cdot P(k)
$$

where $\mathrm{X}_{r}(k)$ is the reconstructed signal, $S=\left\{s_{m n}\right\}$ is an $M \times N$ matrix and $s_{m n}$ is the gain by which the $n$th activation coefficient is transferred to the $m$ th muscle activation signal. Each column of $S$ is referred to as a synergy and the matrix $S$ will be indicated as the synergy matrix.

\section{EMG preprocessing}

The EMG signals were off-line band-pass filtered (fourth-order zero-lag Butterworth digital filter, bandwidth $20-400 \mathrm{~Hz}$ ) to attenuate DC offset, motion artifacts, and high-frequency noise (Hermens et al. 1999). The filtered signals were full-wave rectified and low-pass filtered (fourth-order zero-lag Butterworth digital filter, cutoff frequency $1 \mathrm{~Hz}$ ) to obtain the muscle activation patterns. The surface EMG signals recorded from the upper trunk (PEC and LAT) were contaminated by electrocardiogram (ECG) artifact. Thus a reference channel measuring the ECG signal was recorded from the left pectoralis muscle and used in a least mean squares adaptive digital transversal filter (50 taps) (Mesin et al. 2008) to eliminate the ECG artifact from the two contaminated channels.

Tonic EMG levels were observed in some muscles between tasks, presumably acting as joint stabilizers (Suzuki et al. 2001), e.g., ANC and TLO. Only the time intervals corresponding to movements were analyzed. The center instant of a movement was defined as the time instant of maximum angular velocity, as measured from the kinematics data. Each movement was analyzed in an interval ranging from $1 \mathrm{~s}$ before to $1 \mathrm{~s}$ after the center instant.

\section{Kinematics data processing}

The three-dimensional positions of the reflective markers were projected onto the horizontal plane. The upper trunk and limbs were modeled as an interconnected chain of rigid bodies (Cappellini et al. 2006): LAC-RAC for the upper trunk, RAC-LEP for the arm, and LEP-MST for the lower arm. The joint angles of the shoulder 
(LAC-RAC-LEP) and elbow (RAC-LEP-MST) were calculated at each time sample. The joint angle signals were low-pass filtered (fourth-order zero-lag Butterworth digital filter, cutoff frequency 1 $\mathrm{Hz}$ ) to reduce instrumentation noise. The first derivative of the angle signals was calculated to estimate the angular velocity. The processing of kinematics variables was performed exclusively for the purpose of signal segmentation.

\section{Methods for synergy extraction}

An NMF algorithm (Lee and Seung 2001) was used to extract $N$ muscle synergies from the EMG signals. The synergy matrix $S(M \times N)$ and the activation signal matrix $P(N \times K)$, where $K$ is the number of samples, were initialized with random nonnegative values. The same synergy matrix $S$ was used to describe the muscular activation pattern of a large set of movements, including movements not used for synergy extraction. This was accomplished by a modified version of the NMF algorithm, referred to in the following text as nonnegative reconstruction (NNR). With NNR, the synergy matrix $S$ was fixed and, at each iteration, only the activation signal matrix $P$ was updated, following the update multiplicative rule proposed by Lee and Seung (2001). In contrast to the pseudoinverse approach, which would also provide an estimation of $P$ given $X$ and $S$, the proposed NNR algorithm ensures nonnegative activation signals.

The proposed NNR method is consistent with a practical application in which one synergy matrix is fixed and used to transform activation signals into muscular activation signals. Like other matrix factorizations algorithms, the results of NMF and NNR present ambiguities of scaling and permutation. The scaling ambiguity implies that it is possible to identify only the relative, and not the absolute, activations between synergistic muscles. In this study, the scaling ambiguity has been addressed by normalizing each synergy with respect to the level of activity of the most active muscle in that synergy. With this normalization, the maximum value in each column of the synergy matrix is equal to 1 . The ambiguity of permutation is not relevant in the synergy context. Given a certain synergy matrix as input to the NNR algorithm, the control signal corresponding to the $n$th synergy (the $n$th column in the synergy matrix) is multiplied by the $n$th row of the activation signal matrix.

In this study, we propose methods to extract a common synergy matrix $S$ that describes the entire set of investigated functional tasks in the horizontal plane. The estimated muscular activation pattern $X_{r}=$ $S P$ was compared with the recorded pattern $X$ by the coefficient of determination $r^{2}$. The coefficient of determination used in this study was the square of the correlation coefficient of the concatenated muscular signals. d'Avella et al. $(2003,2006)$ defined the multivariate $r^{2}$ as the variation that can be explained by the model: VAF $=1-$ SSE/SST, where SSE (sum of squared errors) is the unexplained variation and SST (total sum of squares) is the total variation (of the data). The choice adopted in the present study ensures nonnegativity of the performance index. Results obtained with other indexes, such as the VAF index, did not substantially differ from those obtained with the square of the correlation coefficient (see RESULTS).

The reconstruction performance was analyzed as a function of the factorization dimension to select the number of synergies needed to describe the tasks used for synergy calculation. A cross-validation procedure was used in which a random portion of the samples in a data set $(80 \%)$ was selected for synergy extraction and reconstruction was performed on the remaining samples (20\%) (d'Avella et al. 2003). Mean values over 10 simulation runs were used for this analysis and the coefficient of determination was displayed as a function of the number of synergies (range: 1-6). The optimal dimensionality was associated with the number of synergies corresponding to a change in slope of the association between coefficient of determination and number of synergies (d'Avella et al. 2003).

To compare pairs of synergies, the scalar product of the two vectors was divided by the product of the norms. This value ranges between
0 and 1 because vectors of synergies are nonnegative. To test for the level of performance that was due to chance, random synergies were generated by random samples from exponential distributions with a mean value of 10 . These distributions were chosen because they are similar to the distributions observed in the analysis of experimental data in previous studies (Tresch et al. 2006). Random activation patterns were also generated from exponential distributions with the same mean value, but signals were low-pass filtered (fourth-order zero-lag Butterworth digital filter, cutoff frequency $1 \mathrm{~Hz}$ ) to match the frequency content of the experimental activation patterns.

Different synergy matrices were computed from different sets of tasks. Each synergy matrix was then tested by its ability of describing the entire set of directions in the multijoint reaching task investigated. Synergy matrices were extracted from: 1 ) the single-joint movements of the elbow and shoulder or 2) a combination of multijoint movements in $1,2,3$, and 12 directions. In each case, the extracted synergy matrix was used to reconstruct the entire set of directions of the multijoint reaching task with the aim of assessing whether this matrix was sufficiently general to describe a large set of conditions.

For the purpose of synergy extraction, muscle activation patterns (EMG envelopes) were resampled by interpolation to a sampling frequency of $40 \mathrm{~Hz}$.

\section{RES U L TS}

Figure 2 shows a representative example of the 12 muscle activation signals and the 2 joint activation signals during 6 elbow movements (single-joint) of a representative subject.

A EMG

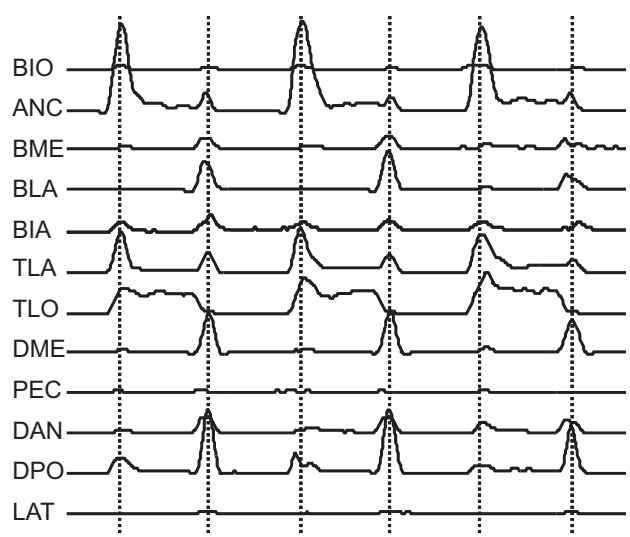

B

Angular

velocity

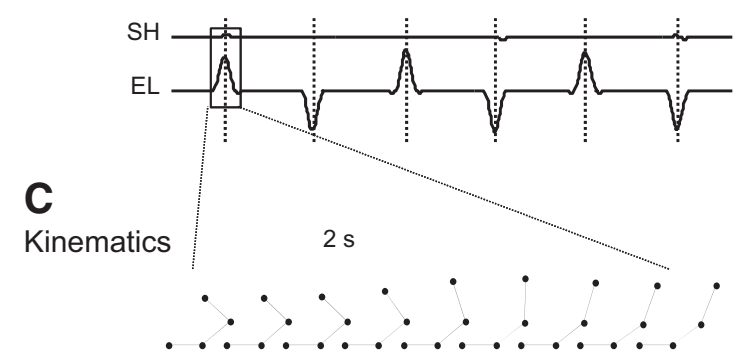

FIG. 2. Representative example of muscle activation patterns ( $A: 12$ traces) and angular velocities $(B)$ of shoulder $(\mathrm{SH})$ and elbow (EL) joints recorded from a subject during 3 repetitions of elbow extension and flexion with the shoulder angle at $135^{\circ}$. The vertical lines indicate the time instants of maximum angular velocity. $C$ : the marker positions of the first movement (indicated by the square in the joint activation patterns) are shown for 9 evenly spaced samples $(250 \mathrm{~ms}$ per interval) over a time interval of $2 \mathrm{~s}$. 
The first movement is illustrated by a chain of rigid bodies. As it appears from this example, the muscle activation pattern is highly redundant. Figure 3 shows the decomposition of the muscle activation pattern represented in Fig. 2 into activation signals and muscle synergies. Synergies were extracted using the NMF algorithm from the signal obtained concatenating the 2 -s intervals centered on the instant of maximum angular velocity of each of the 6 movements $(12 \mathrm{~s}$ in total). Each synergy is activated mainly during either extension or flexion of the elbow; thus the maximum values of the activation signals correspond to the peaks of the joint activation signals for extension and flexion (Fig. 3). The tonic activity level is part of the synergy contributing mainly to elbow extension. In this example, the $r^{2}$ index of reconstruction accuracy was 0.86 .

Figure $4 A$ shows the performance index $r^{2}$ (mean $\pm \mathrm{SD}, n=$ 8 subjects) as a function of dimensionality using NMF when synergies were extracted from the single-joint tasks.

Muscle activation patterns were reconstructed with $r^{2}>0.85$ when using two dimensions in the single-joint movements. Moreover, the performance index steeply increased when in-

\section{Activation signal 1 Synergy 1 Muscle activation patterns}
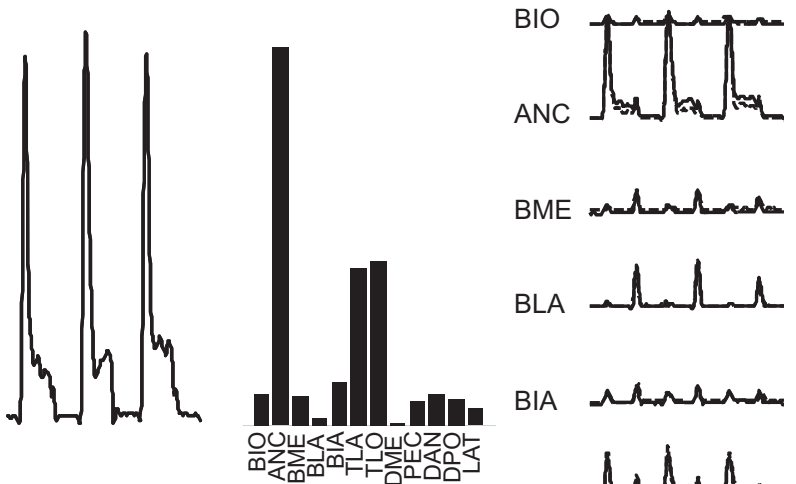

BIA
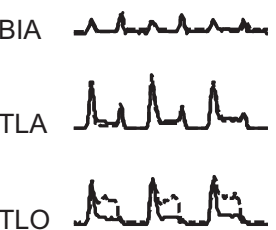

Activation signal 2 Synergy 2
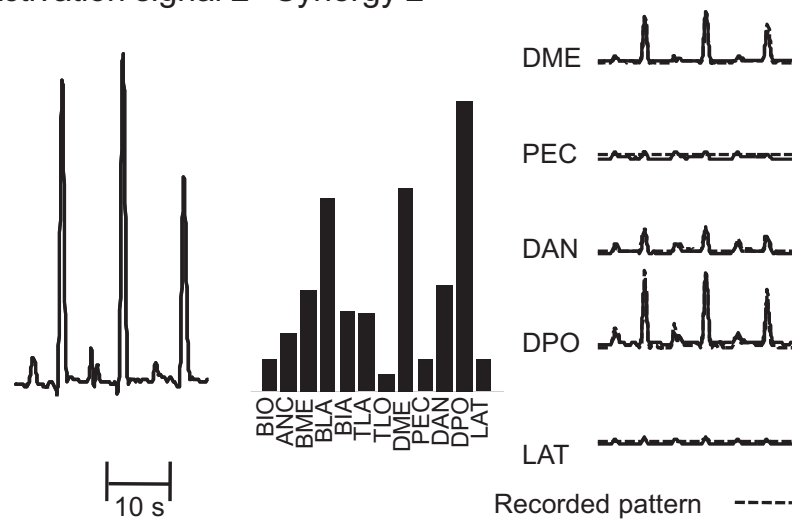

LAT

Recorded pattern

Reconstructed pattern

FIG. 3. Decomposition of the muscle activity pattern shown in Fig. 2 into activation signals and muscle synergies. In all, $12 \mathrm{~s}$ of the signal corresponding to the single-joint movements shown in Fig. 2 were used for the extraction (each of the 6 movements was analyzed in an interval ranging from $1 \mathrm{~s}$ before to $1 \mathrm{~s}$ after the instant of maximum angular velocity). The recorded muscle activation pattern is shown on the right (dashed line) together with the pattern estimated using 2 activation signals (solid line). creasing the number of synergies from 1 to 2 , whereas it did not change substantially for a further increase. Thus it was assumed that the dimensionality of the single-joint muscular activation patterns was two (d'Avella et al. 2003).

The mean similarity between synergies extracted from two repetitions of the same single-joint movement (across 8 subjects and 6 single-joint movements, for a total of 48 comparisons) was 0.99 , indicating high reliability of synergy extraction across repetitions. Conversely, repeating the same analysis on random data led to a mean similarity of 0.67 . Figure $4 B$ shows the synergies extracted from the single-joint movements of shoulder and elbow when the nonmoving joint was fixed at three angles. Considering the task SH2, it can be noted that the most active muscles in the shoulder synergies are the DPO and the DAN (a shoulder extensor and flexor, respectively). During the task EL2, the ANC, which mainly contributes to elbow extension, is the muscle mostly contributing to one of the two synergies, whereas the BME, BLA, and BIA, active during elbow flexion, are grouped in the other synergy. Thus the extraction of synergies from single-joint tasks determined a separation between DOFs. The similarity between synergies extracted from two trials of the same type of movement with the nonmoving joint in different positions was 0.87 (across 8 subjects and 3 combinations for each joint, 72 comparisons in total).

Table 1 shows the reconstruction performance when a muscle activation pattern of one single-joint movement was used to reconstruct another single-joint movement. The performance index was very low when attempting the reconstruction of elbow movements from synergies extracted from shoulder movements and vice versa. However, the performance index $r^{2}$ was high when using synergies obtained from the same movement but with the nonmoving joint at different angles.

The selection of different performance indexes did not change the relative comparisons. For example, the reconstruction of the tasks SH1 and SH2 using synergies extracted from the same tasks led to a performance of $0.76 \pm 0.3$ and $0.87 \pm$ 0.08 , respectively, when using the index VAF (d'Avella et al. 2003). Thus the relative quality of reconstruction was the same as that when analyzed with the index used in this study (Table 1). The trends in the results were also the same when using the two performance indexes for the other tasks.

\section{Reconstruction of multijoint tasks with synergies extracted from single-joint tasks}

Multijoint movements were reconstructed using the synergies extracted from the single-joint movements and synergies directly extracted from a set of multijoint directions. Similar to the results for single-joint tasks, the results presented in the following for the multijoint movements were very similar varying the initial shoulder joint angle of $100^{\circ}, 120^{\circ}$, or $140^{\circ}$. Thus for clarity of the presentation, only the results for the reaching task performed with shoulder joint angle of $120^{\circ}$ are presented in the following.

Table 2 shows the index $r^{2}$ associated with the reconstruction of single-joint and multijoint reaching movements, as well as random structureless data, when using the synergy matrix extracted from single-joint movements. One synergy was not sufficient to describe any task, as predicted by the dimensionality analysis (Fig. 4), whereas two synergies could explain single-joint tasks but not multijoint tasks, as expected. When using four 
A

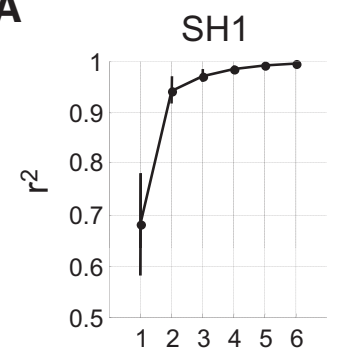

$\mathrm{SH} 2$

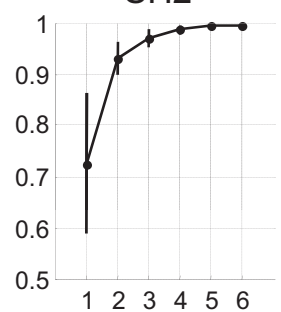

$\mathrm{SH} 3$

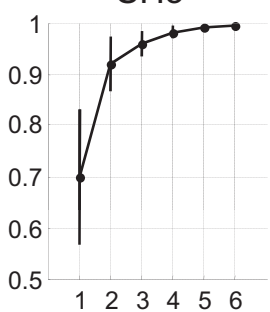

EL1

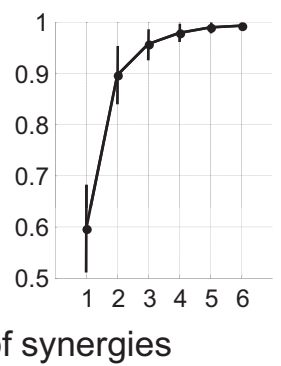

EL2

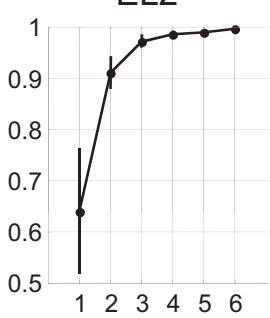

EL3

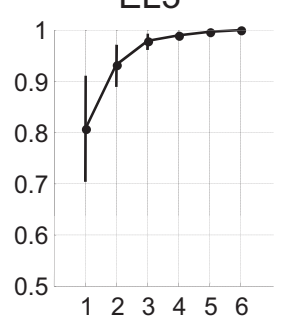

Number of synergies

B
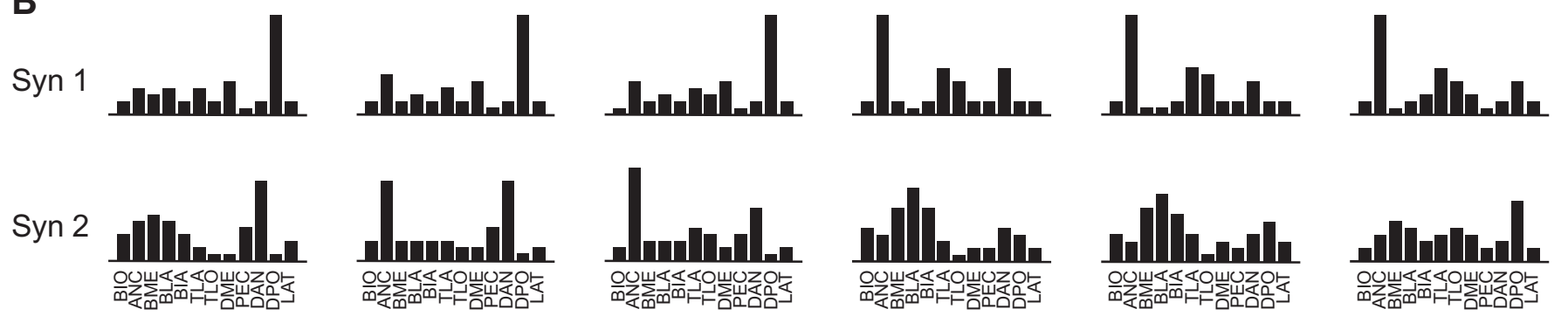

FIG. 4. A: analysis of dimensionality of muscle activation patterns for single-joint tasks. A cross-validation procedure was used to obtain a robust measure of $r^{2}$ for a given dimension. Six data sets were used for synergy extraction (corresponding to the 6 panels): SH1, SH2, SH3 (shoulder movements with elbow at 60,105 , or $150^{\circ}$, respectively) and EL1, EL2, EL3 (elbow movements with the shoulder at 90,135 , or $180^{\circ}$, respectively). Data are mean and SD of $r^{2}$ across the 8 subjects. The number of synergies was estimated as the dimension at which the slope of the $r^{2}$ curve changed sharply (d'Avella et al. 2003) and was 2 in all cases. $B$ : for each of the 6 data sets, the 2 mean synergies (mean across the 8 subjects) are shown. See text for task description.

synergies extracted from the two single-joint tasks independently, the accuracy in the reconstruction was high for both single-joint movements and the reaching task in the horizontal plane. Four random synergies did not allow the description of activation patterns in any condition and random structureless data could not be reconstructed by any of the synergies extracted from the experimental data. Table 2 demonstrates that a large set of directions in a multijoint reaching task can be expressed by one synergy matrix of dimension four, extracted from single-joint tasks and capable of reconstructing a large set of tasks not used for the synergy computation. Moreover, the four synergies extracted using the single-joint tasks were very similar across subjects. The mean similarity of synergies (average across all pairs of subjects) was $0.80 \pm 0.07$.

\section{Directional tuning of synergies extracted from single-joint tasks}

Figure $5 A$ shows the index $r^{2}$ for the 12 targets in the reaching tasks as a circular plot. The reconstruction was performed using the four synergies extracted from the singlejoint movements (two for each single-joint task, extracted independently). In addition, the reconstruction was obtained by using only one synergy, chosen from among those extracted from single-joint movements. The $r^{2}$ index for the multijoint reaching task was $>0.8$ for all directions using the four synergies (Fig. 5). Moreover, the elbow and shoulder contributions were directionally tuned and combined to produce multijoint movements. For example, Fig. 5 indicates that the synergy that group the muscles responsible for shoulder extension (shoulder synergy 1 in Fig. 4B) provided a good reconstruction in the zones of the horizontal plane that can be reached by shoulder extension; similar results are observed for the synergies that group the muscles responsible for shoulder flexion (shoulder synergy 2 in Fig. 4B) and for elbow extension and flexion (elbow synergy 1 and elbow synergy 2). Figure 5 , $B-E$ shows the performance in reconstruction when using three of the four synergies obtained from the single-joint move-

TABLE 1. Performance index in the reconstruction of muscle activation patterns of one movement (indicated in the rows) using two synergies extracted from another movement (indicated in the columns)

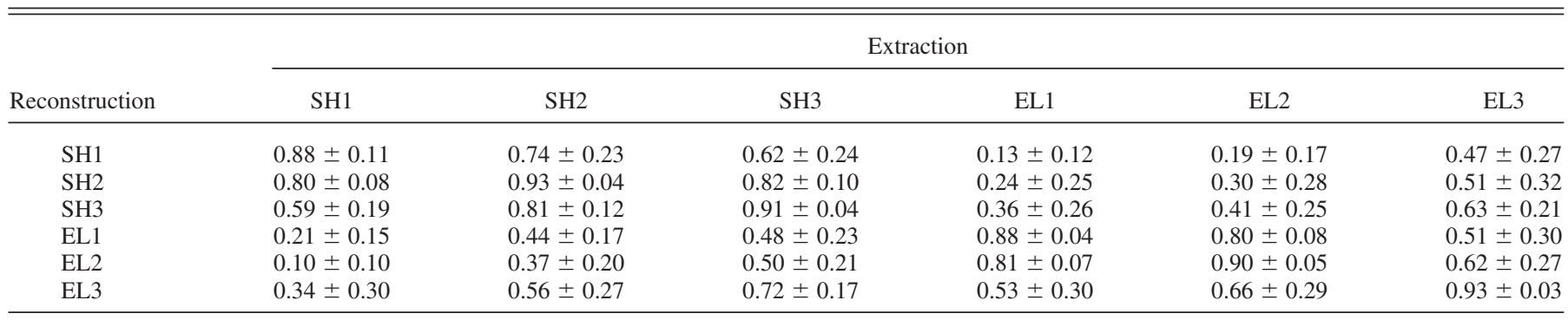

Values are means $\pm \mathrm{SD}$ of $r^{2}, n=8$ subjects. When the extraction pattern and the reconstruction pattern were of the same type (diagonal entries), two different trials were used for extraction and reconstruction. SH1, SH2, SH3: shoulder movements with elbow fixed at 60, 105, or 150 ${ }^{\circ}$; EL1, EL2, EL3: elbow movements with shoulder fixed at 90,135 , or $180^{\circ}$, respectively. See text for task description. 
TABLE 2. Performance index in the reconstruction of muscle activation patterns of one movement (indicated in the rows) using synergies extracted from another movement (indicated in the columns)

\begin{tabular}{lcccccc}
\hline \hline & \multicolumn{5}{c}{ Extraction } \\
\cline { 2 - 7 } Reconstruction & SH2 (1) & EL2 (1) & SH2 (2) & EL2 (2) & SH2 (2) + EL2 (2) & Four Random Synergies \\
\hline SH2 & $0.70 \pm 0.14$ & $0.13 \pm 0.13$ & $0.93 \pm 0.04$ & $0.30 \pm 0.28$ & $0.94 \pm 0.02$ & $0.23 \pm 0.18$ \\
EL2 & $0.12 \pm 0.13$ & $0.62 \pm 0.15$ & $0.37 \pm 0.20$ & $0.90 \pm 0.05$ & $0.92 \pm 0.04$ & $0.21 \pm 0.22$ \\
JO2 & $0.45 \pm 0.17$ & $0.43 \pm 0.16$ & $0.73 \pm 0.10$ & $0.62 \pm 0.18$ & $0.87 \pm 0.03$ & $0.32 \pm 0.22$ \\
Random & $0.01 \pm 0.00$ & $0.01 \pm 0.00$ & $0.06 \pm 0.00$ & $0.07 \pm 0.01$ & $15 \pm 0.03$ & $0.12 \pm 0.01$ \\
\hline
\end{tabular}

Values are means $\pm \mathrm{SD}$ of $r^{2}, n=8$ subjects. In the columns, the number of synergies extracted is denoted in parentheses. When the extraction pattern and

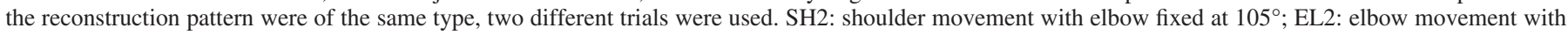
shoulder fixed at $135^{\circ}$; JO2: multijoint reaching tasks (average over the 12 targets). See text for task description.

ments. None of the selections of three synergies allowed accurate reconstruction of the reaching task in all directions. For example, when removing the synergy characterized by a high contribution of the DPO muscle (shoulder synergy 1 in Fig. 4B), the reconstruction worsened in the zone of the horizontal plane that is reached by shoulder extension (Fig. 5B). Similarly, three synergies obtained as linear combinations of the four synergies extracted from the single-joint movements did not allow accurate reconstruction of the multijoint reaching task (results not shown).

These results indicate that if synergies are extracted independently from single-joint movements to describe multijoint tasks, two synergies are needed for each DOF.

\section{Reconstruction of multijoint movements with synergies extracted from multijoint movements}

Synergies were also extracted directly from a set of multijoint directions and used to describe all reaching directions. When four synergies were extracted from only one reaching direction, it was possible to describe that specific direction but it was not possible to accurately describe all the other directions (Fig. 6). Therefore these synergies are specific: the reconstruction of the same task used for extraction has high accuracy, although the reconstruction of other tasks may be poor. The same conclusion was reached when using three synergies extracted from one task only, which allowed reconstruction of only a few directions.

It was also not possible to describe all the reaching directions when using two reaching directions for the synergy extraction, regardless of the number of synergies used (results not shown). However, three synergies extracted from three directions were often sufficient for describing all the other directions with a level of accuracy similar to that obtained when using four synergies extracted from the single-joint movements. The results depended strongly on the directions chosen for synergy extraction. Figure 7 shows some representative examples of quality in task reconstruction when using three reaching directions for synergy extraction and two to four synergies. The choice adopted in Fig. 7, $A$ and $B$ provided good reconstruction for all the directions (if at least three synergies were used), whereas the choice adopted in Fig. 7, $C$ and $D$ led to poor reconstruction in correspondence of the same targets. Note that two of the targets used for synergy extraction in Fig. 7, C and $D$ are close to each other. In general, when the three directions chosen for synergy extraction were sufficiently separated in the horizontal plane, the muscular activation pattern could be well described in all directions by the same matrix comprising three synergies, but not two (Fig. 7, $A$ and $B$ ). However, when the tasks chosen corresponded to similar directions (Fig. 7, $C$ and $D$ ), the muscular activation pattern could not be well described.

Figure 8 shows the index $r^{2}$ averaged over all directions and across the eight subjects when using three synergies extracted from all combinations of three directions. Because Fig. 7 representatively shows for only a few selections of the directions, Fig. 8 indicates that the performance in reconstruction depended substantially on the selection of directions. Table 3 reports the angles between the three directions chosen for synergy extraction that led to the best averaged reconstruction quality when using three synergies. Although the two angles varied depending on the initial direction chosen, on average they were about $120^{\circ}$ (Table 3 ), which indicates the maximum separation between the three directions. When the optimal directions were chosen, three synergies extracted from three directions could describe the muscular activation patterns in all directions, including directions not used for the synergy computation, with reconstruction quality $>0.9$ (Table 3 ). Finally, the three synergies extracted were very similar across subjects for the optimal directions shown in Table 3. The mean similarity of synergies across subjects (average over all pairs of subjects) was $0.76 \pm 0.09$.

\section{I S C U S S I O N}

The study shows that the muscular activation pattern during reaching in the horizontal plane can be characterized by a minimum of three activation signals. The synergy matrix that translates the activation signals into the muscular activation pattern can be obtained from a limited set of single-joint or multijoint movements and allows representation of all reaching tasks. This matrix constitutes a reduction in dimensionality that can be adopted in systems for restoring functions (e.g., FES), by reducing the dimensionality of the control of complex functions.

The single-joint movements could not be described using only one synergy $\left(r^{2}<0.70\right)$. Two synergies, however, were sufficient for describing single-joint movements with performance index about 0.9 , as a result of the nonnegative constraint in muscle activations. The mean muscle synergies across the eight subjects for the single-joint movements indicated that one synergy contributed mainly to either flexion or extension. The mean synergies extracted from shoulder and elbow movements were very different and almost orthogonal (null scalar product), indicating a separation between the two DOFs. 
A

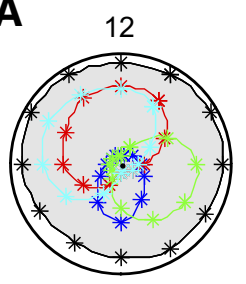

* 1 synergy (sh ext)

1 synergy (sh flex)

* 1 synergy (el ext)

* 1 synergy (el flex)

* all 4 synergies

B

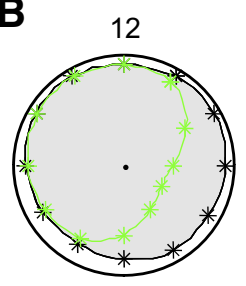

* 3 synergies

(el ext, el flex, sh flex)

* all 4 synergies

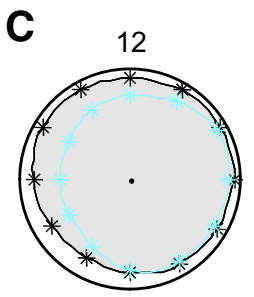

3 synergies

(el ext, el flex, sh ext)

* all 4 synergies

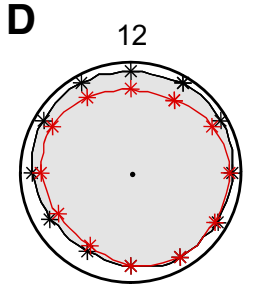

\section{* 3 synergies}

(el flex, sh ext, sh flex)

* all 4 synergies

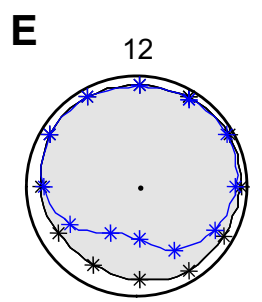

* 3 synergies

(el ext, sh ext, sh flex)

* all 4 synergies

FIG. 5. Circular plots representing the performance index value when describing the muscular patterns during reaching tasks in the 12 directions. The values are averages across 8 subjects. The central black dot denotes no reconstruction $\left(r^{2}=0\right)$, the external circumference (solid line) denotes perfect reconstruction $\left(r^{2}=1\right)$, whereas the black star symbols indicate the performance index obtained when using the 4 synergies extracted from the singlejoint movements. $A$ : the muscular patterns are described using only one of the 4 synergies extracted from single-joint movements (green: shoulder extension; cyan: shoulder flexion; red: elbow extension; blue: elbow flexion). $B$ : same as in $A$, but using only 3 of the 4 synergies (elbow extension, elbow flexion, and shoulder flexion). $C$ : as in $A$, using elbow extension, elbow flexion, and shoulder extension. $D$ : as in $A$, using elbow flexion, shoulder extension, and shoulder flexion. $E$ : as in $A$, using elbow extension, shoulder extension, and shoulder flexion.

The kinematics of movements in a multijoint system can be represented as a combination of single-joint movements. The results of this study showed that the muscular activation patterns can also be well reconstructed (mean $r^{2}=0.87$ ) using two synergies extracted from each single-joint movement (four in total). The extraction of synergies from single-joint tasks provided the way to describe the multijoint tasks in a subspace of orthogonal synergies associated with the two DOFs of the tasks. When using these orthogonal set of synergies, it was not possible to decrease the dimension to fewer than four. Three synergies extracted directly from the single-joint movements or from linear combinations of synergies extracted from the single-joint movements were not sufficient to describe all directions. Thus the subspace spanned by orthogonal synergies extracted from the two DOFs had a dimension of four when used to describe the multidirectional reaching task.

The extraction of synergies from each individual reaching direction (Fig. 6) allowed the description of the specific direction with low dimensionality, similar to the use of four synergies from single-joint tasks. However, the synergies extracted from a specific direction could not describe all the reaching directions in the horizontal plane and were specific to directions similar to those used for the extraction (Fig. 6). These synergies would not allow an efficient reduction in the complexity of the control since a different synergy matrix would be required for different directions. To obtain a synergy matrix that characterized all directions, it was necessary to extract the synergy space from a representative set of directions. When at least three representative directions were included in the procedure for synergy extraction, it was possible to reconstruct all reaching directions with only three synergies and thus less than that in the case of orthogonal synergies (Fig. 7, $A$ and $B$ ). However, in this case the choice of the tasks from which the
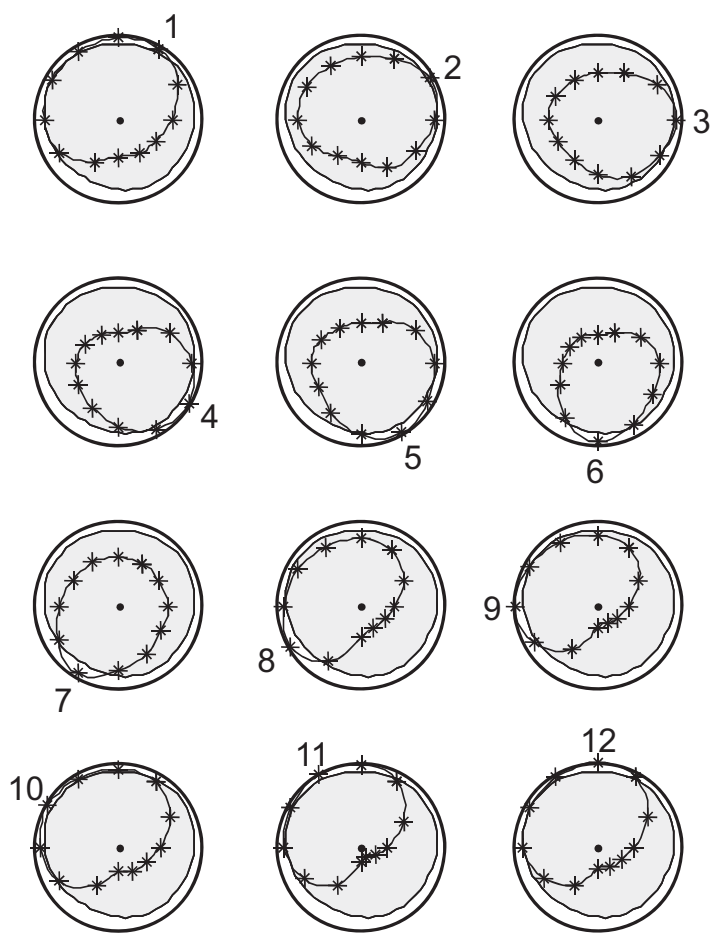

FIG. 6. Circular plots representing the performance index value when describing the muscular patterns (star symbols) during reaching tasks in the 12 directions. Four synergies were extracted from each direction and these synergies were used to describe all tasks. The task from which the 4 synergies were extracted is indicated by the corresponding number (1 to 12) along the circumference. The central black dot denotes no reconstruction $\left(r^{2}=0\right)$, the external circumference (solid line) denotes perfect reconstruction $\left(r^{2}=1\right)$, whereas the gray shaded area (delimitated by solid line) indicates the performance index obtained when using the same set of 4 synergies extracted from the single-joint movements for reconstructing all reaching tasks. The synergies obtained from one reaching movement only are context-specific and they do not allow good reconstruction of all tasks. 
A

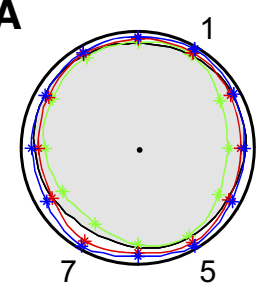

B

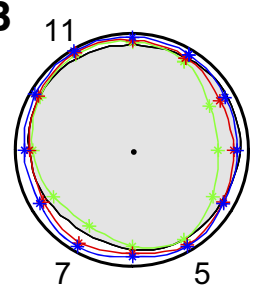

C
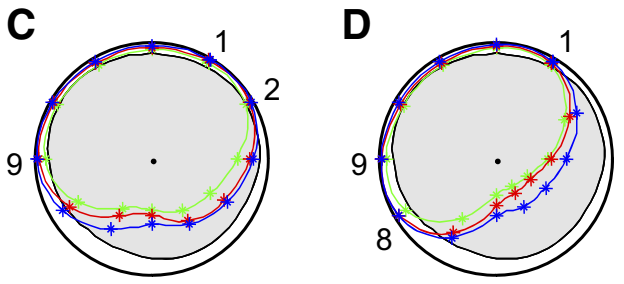

2 synergies $\quad * 3$ synergies $* 4$ synergies

FIG. 7. Circular plots representing the performance index value when describing the muscular patterns during reaching tasks in the 12 directions. Two (green lines), 3 (red lines), and 4 (blue lines) synergies were extracted from 3 of the reaching directions and these synergies were used to describe all directions. The directions from which the synergies were extracted are indicated by the corresponding numbers ( 1 to 12 ) along the circumference. Four representative examples are shown for different choices of the 3 directions. The central black dot denotes no reconstruction $\left(r^{2}=0\right)$, the external circumference (solid line) denotes perfect reconstruction $\left(r^{2}=1\right)$, whereas the gray shaded area (delimitated by solid line) indicates the performance index obtained when using the same set of 4 synergies extracted from the single-joint movements for reconstructing all reaching tasks. The reconstruction quality strongly depended on the 3 directions chosen for synergy extraction.

synergies were computed was important for the accuracy of the reconstruction (Figs. 7 and 8). As was shown in Fig. 7, there were several choices for three directions from where synergies could be extracted to accurately describe the entire set of directions. The three optimal directions were on average equiseparated, i.e., the angles between directions were about $120^{\circ}$

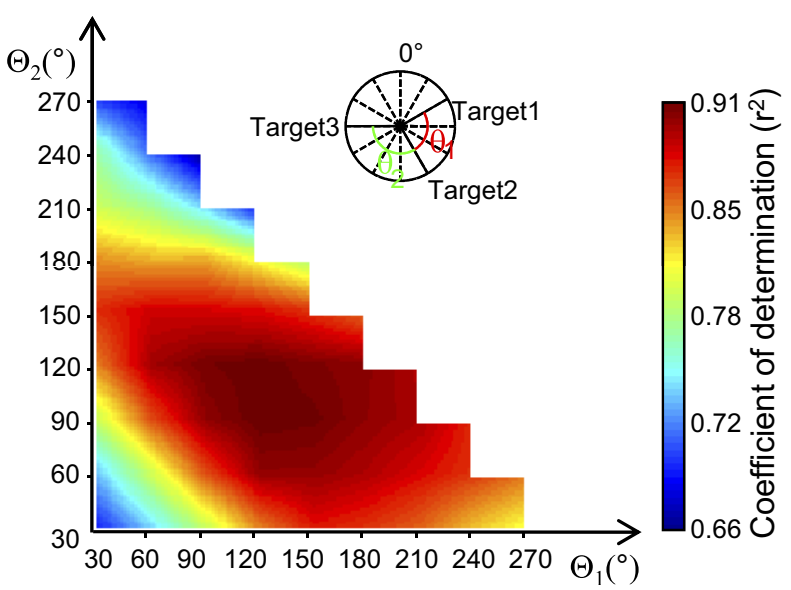

FIG. 8. Colored map (interpolated by a factor 4) representing the performance index value when describing the muscular patterns using 3 synergies extracted from 3 of the reaching directions. Given a reference target (target 1 in the inset), a pair of angles $\theta_{1}$ and $\theta_{2}$ define 3 directions (see inset). For each pair of angles $\theta_{1}$ and $\theta_{2}$ there are 12 choices of the reference target (target 1 ). The $r^{2}$ value in reconstruction was computed when the synergies were extracted from the 3 directions defined by $\theta_{1}$ and $\theta_{2}$ for all choices of target 1 (12 values of $r^{2}$ for each pair of angles $\theta_{1}$ and $\theta_{2}$ ). The map represents, for each pair $\theta_{1}$ and $\theta_{2}$, the value of $r^{2}$ averaged over the 12 choices of target 1 . Results from the 8 subjects are also averaged in this representation.
TABLE 3. Set of directions leading to the best average reconstruction quality for the 12 choices of target 1

\begin{tabular}{cccc}
\hline \hline Target 1 & $\theta_{1}$, deg & $\theta_{2}$, deg & $r^{2}$ \\
\hline 1 & 120 & 60 & 0.90 \\
2 & 90 & 180 & 0.90 \\
3 & 90 & 150 & 0.90 \\
4 & 90 & 120 & 0.90 \\
5 & 60 & 120 & 0.91 \\
6 & 150 & 120 & 0.90 \\
7 & 120 & 180 & 0.91 \\
8 & 150 & 120 & 0.90 \\
9 & 120 & 120 & 0.90 \\
10 & 210 & 60 & 0.90 \\
11 & 180 & 60 & 0.91 \\
12 & 150 & 90 & 0.90 \\
Mean \pm SD & $127.5 \pm 42.7$ & $115.0 \pm 42.1$ & $0.90 \pm 0.00$
\end{tabular}

See inset in Fig. 8 for definition of target 1. Three synergies were extracted from three directions and used to reconstruct the reaching task in all directions. The first direction (target 1) was fixed between 1 and 12 (first column), the second was separated from the first by the angle $\theta_{1}$, and the third from the second the angle $\theta_{2}$ (see inset in Fig. 8). The values of the performance index, averaged over the reconstruction of all 12 directions, are reported for the set of directions leading to the best average reconstruction quality for the 12 choices of the first direction. The performance index was similar for the optimal set of three directions independently of the first direction. The mean and SD of the angles between the optimal directions are also reported.

(Table 3). The reconstruction quality did not substantially depend on the rotation of the three directions as long as the directions were approximately equiseparated (Table 3 ). The observation that any combination of the three synergies extracted from single-joint movements did not allow accurate reconstruction in all the directions indicates that at least one synergy extracted from multijoint tasks was different with respect to the synergies extracted from single-joint tasks.

Both the single- and multijoint synergy identification used in our study provided a sufficient basis set to explain the variance in muscle activation patterns during planar reaching movements. Since these synergies also showed high intersubject similarity, they are suitable for applicative purposes, such as FES. Reduction in the number of control signals obtained by extracting synergies from multijoint movements depends on the fact that the two joints are not controlled independently during reaching, which implies that the CNS does not separate the two DOFs.

A definitive physiologically validated notion of "natural synergies" (i.e., encoded by the CNS) has not been obtained. However, evidence so far indicates that natural modules operate over multiple joints [e.g., synergies associated with spinal force fields in the frog (Giszter et al. 1993) as well as synergies in human reaching (d'Avella et al. 2006; Iftime et al. 2005)]. Previous studies on spatial synergies (Iftime et al. 2005) showed a strong coupling of shoulder flexion/extension and elbow flexion/extension for reaching. d'Avella et al. (2006) also pointed out that synergies acting during reaching include anatomically synergistic muscles but also muscles active on different joints. Moreover, animal experiments also indicate that movement modules involve multiple joints, since all observed force fields have rather complex structures that cannot be obtained by single-joint torque profiles (Giszter et al. 1993).

In our experiment, the fact that the set of three synergies from multijoint movements reconstructed the data with the same accuracy as that of the set of four synergies from 
single-joint movements suggests that the synergies extracted from single-joint movements are not the ones encoded by the CNS. Rather, single-joint extraction forces the algorithm to find synergies specialized in describing the biomechanical demand of single-joint movements, excluding system dynamics that are not relevant to single-joint tasks, i.e., excluding the coupling that naturally occurs during multijoint reaching. In other words, the data set used for single-joint synergy extraction is not sufficient to capture the invariant spatial structure underlying multijoint reaching tasks with minimal dimensionality. Indeed, such synergies are specific to the context of single-joint movements. Generalizing to other tasks in which more degrees of freedom are involved, the data set used for synergy extraction has to include multijoint movements representative of the degrees of freedom at those joints to capture the coupling between the degrees of freedom.

Ajiboye and Weir (2009) recently observed that 8 muscle synergies extracted from a set of 11 static hand postures could predict the EMG pattern of 11 hand muscles for 22 postures. In that study, a decrease in the dimensionality to $<8$ resulted in a loss of similarity of the synergies across the subject population. However, the use of muscle synergies as a basis of control for movement restoration is relevant only if an effective reduction in the control complexity can be obtained. Our study demonstrated that it is possible to control 12 muscles with only three control signals to produce a large repertoire of multijoint movements in the horizontal plane with a transformation matrix that is common to all tasks and similar across subjects.

In this study, the level of muscle activity has been estimated from the amplitude of the surface EMG. Although this relation is not exact (Clancy et al. 2002; Farina et al. 2004) and there is variability across subjects due to electrode placements, subcutaneous layer thickness, and muscle size, it is sufficient for the purposes of the study, as discussed in previous works (d'Avella et al. 2003, 2006). Among the factors affecting the results, EMG cross talk deserves a special attention when extracting muscle synergies (d'Avella et al. 2008). Cross talk between muscles may appear as synergistic muscle behavior and was not accounted for in the synergy model. Although it is not currently possible to eliminate or accurately identify EMG cross talk (Farina et al. 2004), d'Avella et al. (2006, 2008) previously demonstrated during similar tasks as those analyzed in this study that the extracted synergies do not substantially change when eliminating from the analysis the muscles mostly affected by potential cross talk.

This study focused on reaching tasks, although the paradigm of muscle synergies could also be effectively exploited in the restoration of lower-limb muscle activations during posture or locomotion. For example, Torres-Oviedo and Ting (2007) investigated postural responses to multidirectional perturbations and concluded that a limited number of synergies robust across trials and subjects sufficed for reproducing leg and lower-back muscle activation patterns (see also Torres-Oviedo et al. 2006). Raasch and Zajac (1999) showed in simulations and human experiments that pedaling with different cadences, workloads, and directions can be performed with the same functional muscle groups. A small number of activation signals also account for the muscle activity patterns during locomotion (Ivanenko et al. 2004; Olree and Vaughan 1995).

In conclusion, a large set of multijoint reaching movements in the horizontal plane can be described to a good approxima- tion by linear combinations of a limited number (at least three) of activation signals that are sufficient to generate the activation patterns of a large set of muscles. The coefficients in the linear combination (synergy matrix) can be fixed for all reaching directions and thus represent an effective transformation matrix valid for a large class of movements and similar across subjects. This property is fundamental for a practical implementation of the synergy approach in techniques for restoration of functions.

\section{G R A N T S}

This study was partly sponsored by European Project TREMOR under Contract 224051 to D. Farina.

\section{REFERENCES}

Ajiboye AB, Weir RF. Muscle synergies as a predictive framework for the EMG patterns of new hand postures. J Neural Eng 6: 036004, 2009.

Barbeau H, McCrea DA, O'Donovan MJ, Rossignol S, Grill WM, Lemay MA. Tapping into spinal circuits to restore motor function. Brain Res Rev 30: 27-51, 1999.

Berniker M, Jarc A, Bizzi E, Tresch MC. Simplified and effective motor control based on muscle synergies to exploit musculoskeletal dynamics. Proc Natl Acad Sci USA 106: 7601-7606, 2009.

Bernstein N. The Coordination and Regulation of Movements. New York: Pergamon Press, 1967.

Bizzi E, Cheung VC, d'Avella A, Saltiel P, Tresch M. Combining modules for movement. Brain Res Rev 57: 125-133, 2008.

Bizzi E, d'Avella A, Saltiel P, Tresch M. Modular organization of spinal motor systems. Neuroscientist 8: 437-442, 2002.

Bizzi E, Mussa-Ivaldi FA, Giszter S. Computations underlying the execution of movement: a biological perspective. Science 253: 287-291, 1991.

Boyce VS, Lemay MA. Modularity of endpoint force patterns evoked using intraspinal microstimulation in treadmill trained and/or neurotrophin-treated chronic spinal cats. J Neurophysiol 101: 1309-1320, 2009.

Cappellini G, Ivanenko YP, Poppele RE, Lacquaniti F. Motor patterns in human walking and running. J Neurophysiol 95: 3426-3437, 2006.

Clancy EA, Morin EL, Merletti R. Sampling, noise-reduction and amplitude estimation issues in surface electromyography. J Electromyogr Kinesiol 12: $1-16,2002$.

d'Avella A, Bizzi E. Shared and specific muscle synergies in natural motor behaviors. Proc Natl Acad Sci USA 102: 3076-3081, 2005.

d'Avella A, Fernandez L, Portone A, Lacquaniti F. Modulation of phasic and tonic muscle synergies with reaching direction and speed. $J$ Neurophysiol 100: 1433-1454, 2008.

d'Avella A, Portone A, Fernandez L, Lacquaniti F. Control of fast-reaching movements by muscle synergy combinations. J Neurosci 26: 7791-7810, 2006.

d'Avella A, Saltiel P, Bizzi E. Combinations of muscle synergies in the construction of a natural motor behavior. Nat Neurosci 6: 300-308, 2003.

De Luca CJ, Erim Z. Common drive in motor units of a synergistic muscle pair. J Neurophysiol 87: 2200-2204, 2002.

Ethier C, Brizzi L, Darling WG, Capaday C. Linear summation of cat motor cortex outputs. J Neurosci 26: 5574-5581, 2006.

Farina D, Merletti R, Enoka RM. The extraction of neural strategies from the surface EMG. J Appl Physiol 96: 1486-1495, 2004.

Gabriel DA. Shoulder and elbow muscle activity in goal-directed arm movements. Exp Brain Res 116: 359-366, 1997.

Galloway JC, Koshland GF. General coordination of shoulder, elbow and wrist dynamics during multijoint arm movements. Exp Brain Res 142: 163-180, 2002.

Giszter SF, Mussa-Ivaldi FA, Bizzi E. Convergent force fields organized in the frog's spinal cord. J Neurosci 13: 467-491, 1993.

Gottlieb GL, Song Q, Hong DA, Corcos DM. Coordinating two degrees of freedom during human arm movement: load and speed invariance of relative joint torques. J Neurophysiol 76: 3196-3206, 1996.

Hermens HJ, Freriks B, Merletti R, Hägg GG, Stegeman D, Blok J, Rau G, Disselhorst-Klug C. European Recommendations for Surface ElectroMyoGraphy: Results of the SENIAM Project. Enschede, The Netherlands: Roessingh Research and Development, 1999. 
Hincapie JG, Kirsch RF. EMG-based control for a C5/C6 spinal cord injury upper extremity neuroprosthesis. Conf Proc IEEE Eng Med Biol Soc 2007: 2432-2435, 2007.

Hincapie JG, Kirsch RF. Feasibility of EMG-based neural network controller for an upper extremity neuroprosthesis. IEEE Trans Neural Syst Rehabil Eng 17: 80-90, 2009.

Holdefer RN, Miller LE. Primary motor cortical neurons encode functional muscle synergies. Exp Brain Res 146: 233-243, 2002.

Iftime SD, Egsgaard LL, Popovic MB. Automatic determination of synergies by radial basis function artificial neural networks for the control of a neural prosthesis. IEEE Trans Neural Syst Rehabil Eng 13: 482-489, 2005.

Ivanenko YP, Poppele RE, Lacquaniti F. Five basic muscle activation patterns account for muscle activity during human locomotion. J Physiol 556: 267-282, 2004.

Kandel ER, Schwartz JH, Jessell TM. Principles of Neural Science (4th ed.). New York: McGraw-Hill Medical, 2000.

Lacquaniti F, Soechting JF. Coordination of arm and wrist motion during a reaching task. J Neurosci 2: 399-408, 1982.

Lee DD, Seung HS. Algorithms for non-negative matrix factorization. $A d v$ Neural Inform Process Syst 13: 556-562, 2001.

Lemay MA, Galagan JE, Hogan N, Bizzi E. Modulation and vectoria summation of the spinalized frog's hindlimb end-point force produced by intraspinal electrical stimulation of the cord. IEEE Trans Neural Syst Rehabil Eng 9: 12-23, 2001.

Lemay MA, Grill WM. Modularity of motor output evoked by intraspinal microstimulation in cats. J Neurophysiol 91: 502-514, 2004.

Li ZM. Functional degrees of freedom. Motor Control 10: 301-310, 2006.

Mesin L, Boye A, Troiano A, Merletti R, Farina D. Real-time reduction of power line interference in m-channel surface EMG. In: Proceedings of the XVIIth Congress of the International Society of Electrophysiology and Kinesiology (ISEK), June 18-21, 2008, Niagara Falls, Canada. Amsterdam: Elsevier Science, 2008.

Mussa-Ivaldi FA. From basis functions to basis fields: vector field approximation from sparse data. Biol Cybern 67: 479-489, 1992.
Mussa-Ivaldi FA, Giszter SF. Vector field approximation: a computational paradigm for motor control and learning. Biol Cybern 67: 491-500, 1992.

Mussa-Ivaldi FA, Giszter SF, Bizzi E. Linear combinations of primitives in vertebrate motor control. Proc Natl Acad Sci USA 91: 7534-7538, 1994.

Olree KS, Vaughan CL. Fundamental patterns of bilateral muscle activity in human locomotion. Biol Cybern 73: 409-414, 1995.

Popovic MB, Popovic DB, Sinkjaer T, Stefanovic A, Schwirtlich L. Restitution of reaching and grasping promoted by functional electrical therapy. Artif Organs 26: 271-275, 2002.

Raasch CC, Zajac FE. Locomotor strategy for pedaling: muscle groups and biomechanical functions. J Neurophysiol 82: 515-525, 1999.

Saltiel P, Wyler-Duda K, d'Avella A, Tresch MC, Bizzi E. Muscle synergies encoded within the spinal cord: evidence from focal intraspinal NMDA iontophoresis in the frog. J Neurophysiol 85: 605-619, 2001.

Suzuki M, Shiller DM, Gribble PL, Ostry DJ. Relationship between cocontraction, movement kinematics and phasic muscle activity in single-joint arm movement. Exp Brain Res 140: 171-181, 2001.

Ting LH, Macpherson JM. A limited set of muscle synergies for force control during a postural task. J Neurophysiol 93: 609-613, 2005.

Ting LH, McKay JL. Neuromechanics of muscle synergies for posture and movement. Curr Opin Neurobiol 17: 622-628, 2007.

Torres-Oviedo G, Macpherson JM, Ting LH. Muscle synergy organization is robust across a variety of postural perturbations. J Neurophysiol 96: 1530-1546, 2006.

Torres-Oviedo G, Ting LH. Muscle synergies characterizing human postural responses. J Neurophysiol 98: 2144-2156, 2007.

Tresch MC, Cheung VC, d'Avella A. Matrix factorization algorithms for the identification of muscle synergies: evaluation on simulated and experimental data sets. J Neurophysiol 95: 2199-2212, 2006.

Tresch MC, Saltiel P, Bizzi E. The construction of movement by the spinal cord. Nat Neurosci 2: 162-167, 1999.

Weiss EJ, Flanders M. Muscular and postural synergies of the human hand. J Neurophysiol 92: 523-535, 2004. 